\title{
New horizons in multimodality molecular imaging and novel radiotracers
}

\author{
Authors: Sally Barrington, ${ }^{\mathrm{A}}$ Philip Blower ${ }^{\mathrm{B}}$ and Gary Cook $^{\mathrm{C}}$
}

Positron emission tomography (PET)/computerised tomography is now established in clinical practice for oncologic and non-oncological applications. Improvement and development of scanner hardware has allowed faster acquisitions and wider application. PET/magnetic resonance imaging offers potential improvements in diagnostic accuracy and patient acceptability but clinical applications are still being developed. A range of new radiotracers and non-radioactive contrast agents is likely to lead to a growth in hybrid molecular imaging applications that will allow better characterisation of disease processes.

\section{Introduction}

Molecular and hybrid imaging, particularly positron emission tomography/computerised tomography (PET/CT), is now an established imaging method used in clinical practice. However, the clinical indications for PET/CT continue to expand and novel hybrid imaging methods, such as PET/magnetic resonance imaging (PET/MRI), and novel imaging probes continue to be developed and adopted into clinical practice.

\section{$\mathrm{PET} / \mathrm{CT}$}

PET/CT has come of age since it was hailed as the 'medical invention of the year 2000' by Time Magazine, combining functional and anatomical information in a single scanning session.

PET/CT has mainly been used in oncology, with increased glucose metabolism occurring in most cancers, imaged with 18F-fluorodeoxyglucose (FDG). ${ }^{1}$ FDG uptake occurs even in normal sized lymph nodes with tumour involvement, in bone marrow and some organs where CT can be less sensitive and where MRI has mostly provided regional rather than wholebody assessment. Furthermore, FDG uptake differentiates viable tumour from fibrosis after treatment and FDG uptake changes faster during treatment than tumours change in size. PET/CT thus enables better (re)staging, assessment of relapse

Authors: ${ }^{A}$ professor of PET imaging, King's College London and

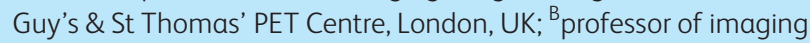
chemistry, King's College London, London, UK; 'professor of PET imaging, Department of Cancer Imaging and Guy's \& St Thomas' PET Centre, King's College London, London, UK and earlier evaluation of treatment success or failure than is possible using CT or MRI in many cancers. PET/CT is now being used to tailor treatment according to individual response to chemotherapy in Hodgkin lymphoma - one of the first examples of 'personalised medicine' to reach the clinic., ${ }^{2,3}$ Suspected lung cancer, including characterisation of lung nodules (which are common in patients with pulmonary disease), is a common indication for PET/CT where biopsy may be challenging. ${ }^{1} \mathrm{UK}$ evidence-based guidelines used for commissioning of NHS scans recommend PET/CT for

\section{Key points}

$\mathrm{PET} / \mathrm{CT}$ is widely used in oncology to (re)stage, assess relapse and response to treatment in many cancers using FDG, with advantages over $\mathrm{CT}$ and MRI alone

The use of PET/CT is not limited to oncology, with funded applications in infection and inflammation, to detect focal sepsis in challenging cases, in the assessment of vasculitis, suspected cardiac sarcoidosis, cardiac hibernation and in evaluation of dementias

PET/MRI has the potential to replace some indications that are currently imaged with PET/CT, particularly in areas where both PET and MRI data are required, where MRI is already superior to $\mathrm{CT}$ and where minimising radiation dose to the patient is particularly important

PET and MRI combined have the potential to be synergistic, eg 'smart' MRI contrast agents are in principle able to measure regional molecular changes (eg $\mathrm{pH}$, calcium concentration) and could gain utility by the incorporation of a PET radiolabel to measure the regional concentration of the contrast agent

PET in combination with MRI and/or optical contrast imaging has the potential to enhance surgery by allowing detailed presurgical delineation of both structure and molecular function, together with visualisation of diseased tissue during surgery

KEYWORDS: Computerised tomography, contrast agents, magnetic resonance imaging, optical imaging, positron emission tomography 
evaluation of solid solitary pulmonary nodules with an initial risk of malignancy of $>10 \%$ using the Brock model, provided the nodule is large enough for detection $(>8-10 \mathrm{~mm}) .{ }^{4}$

Further tracers are becoming available for cancers not well imaged by FDG, including gallium-68 $\left({ }^{68} \mathrm{Ga}\right)$-labelled somatostatin receptor agents, eg ${ }^{68} \mathrm{Ga}$-dotatate for pulmonary carcinoid and neuroendocrine tumours and ${ }^{68} \mathrm{Ga}$-prostate specific membrane antigen (PSMA) for prostate cancer. The use of PET/CT for 'theranostics' - imaging cancers with one tracer (diagnostics) then replacing the radionuclide with another for therapy - is gaining momentum; this is another example of 'personalised medicine'. ${ }^{5}$ A tumour showing uptake with ${ }^{68} \mathrm{Ga}$-dotatate may be treated with peptide-receptorradionuclide-therapy, replacing ${ }^{68} \mathrm{Ga}$ with a beta-emitting radionuclide such as yttrium-90 or lutetium-177 labelled with dotatate. $^{5}$

PET/CT applications are not limited to oncology. ${ }^{4}$ Infection and inflammation also have enhanced glucose (and FDG) metabolism. PET/CT is a quick alternative method of detecting the source of sepsis in problematic cases, pyrexia of unknown origin (PUO) and, occasionally, suspected infection of vascular grafts or cardiac implantable devices, and does not involve blood labelling.

FDG PET/CT is used in selected patients with suspected vasculitis to determine extent and distribution of disease activity (Fig 1) and to exclude underlying malignancy, as with other conditions that may be paraneoplastic manifestations. ${ }^{4}$ Sarcoidosis is well imaged but FDG PET/CT should be reserved for cases where there is diagnostic uncertainty, eg in suspected cardiac sarcoidosis. Further cardiac applications include assessment of hibernating myocardium in patients with severe ischaemic heart failure to determine whether revascularisation and/or medical management is appropriate using rubidium- 82 or nitrogen-13-ammonia as perfusion agents and FDG to determine myocardial viability.

Dementias have different patterns of FDG uptake and PET/ CT can assist in evaluating memory loss and neurological symptoms in patients with suspected Alzheimer's disease (AD) and fronto-temporal dementias. ${ }^{4,6}$ Newer amyloid tracers have a high negative predictive value for ruling out $\mathrm{AD}$, yet limited specificity. Amyloid tracers detect amyloid deposition, which occurs in normal ageing. They cannot differentiate AD from other types of dementia and are not currently widely funded in the NHS. It is also now possible to image abnormal deposition of tau phosphoproteins with PET, a method that may offer greater specificity in dementia. ${ }^{6}$

$\mathrm{PET} / \mathrm{CT}$ is now routinely used in UK practice with many routine oncological applications as well as a growing list of robust indications in inflammation, neurology and cardiology. Its success has stimulated development of other multimodality molecular imaging, including single-photon emission computerised tomography/CT, PET/MRI and combinations of radionuclide imaging agents with optical contrast.

\section{PET/MRI}

The development of PET/MRI scanners has been slower than $\mathrm{PET} / \mathrm{CT}$, mainly because of difficulties in engineering and the cost of PET detectors that can operate in a magnetic field. However, within the last 5 years, consecutive and simultaneous hybrid PET/MRI scanners have become available and are being evaluated for indications where there may be superiority over PET/CT. Advantages of MRI over CT include superior tissue contrast resolution, no ionising radiation and ability to add function and physiological measurements to morphological scans. Functional MRI includes dynamic contrast enhancement (DCE) with gadolinium contrast agents to measure blood flow ${ }^{7}$ and diffusion-weighted imaging to measure the diffusion of water molecules. Tightly packed cells, eg malignant tumours, exhibit restriction of water molecules with increased signal of diffusion-weighted images and a reduction in the apparent diffusion coefficient. ${ }^{8}$

Most PET/MRI scanners allow simultaneous acquisition of PET and MRI data, in contrast to PET/CT where the two acquisitions are consecutive. The radiation exposure from PET/MRI is approximately half that of PET/CT, a feature that may be important in paediatric scans or in young patients who are likely to require several follow-up scans, eg patients with lymphoma. For patients who require a PET scan and an MRI scan, there is the additional benefit of reducing the number of appointments and hospital visits. Disadvantages of PET/MRI include a greater capital cost, low availability at the current time and the relatively long scan acquisition times (30-60 minutes) compared with PET/CT (20-30 minutes).

While the cost-effectiveness of PET/MRI in most clinical indications has yet to be established, increasing data show clinical efficacy in a number of oncologic applications. For most oncologic indications, PET/MRI shows similar diagnostic performance to PET/CT when MRI is used only as an anatomical framework. Some additional advantage may be available in assessing skeletal metastases and prostate cancer. In contrast, there is a poorer sensitivity compared with PET/CT for detecting pulmonary metastases. ${ }^{9}$ So far, the potential added value of functional capabilities of MRI has not been adequately assessed. However, with increased use of diffusion-weighted imaging, DCE-MRI and magnetic resonance spectroscopy in clinical applications, such as for whole-body skeletal imaging in myeloma ${ }^{10}$ or assessing prostate cancer, ${ }^{11}$ (Fig 2) this will need to be tested.

In the short term, it is expected that PET/MRI will be suitable for patients requiring both PET and MRI scans at similar points in their diagnostic pathway, eg biochemical recurrence of prostate cancer, recurrent brain tumours and investigation of dementia. In addition, PET/MRI may show utility in paediatric imaging, especially to minimise radiation in non-malignant conditions, and in young patients with curable cancer that may require serial imaging, eg lymphoma. As novel, more specific tracers are developed, there may become a greater need to accurately determine the site and anatomical relationships of any abnormal tracer uptake or to define underlying biological abnormalities in a multiparametric method. These are areas where combined PET/MRI would be ideal.

\section{Novel contrast agents}

While clinical use of molecular and metabolic imaging (with radionuclides) in combination with anatomical referencing (by $\mathrm{CT}$ and more recently MRI) is established, there is potential to go beyond the current state of the art to significantly enhance the depth and quality of information and range of applications of combined modality imaging. This can be achieved both by using existing contrast agents and by developing new ones that 

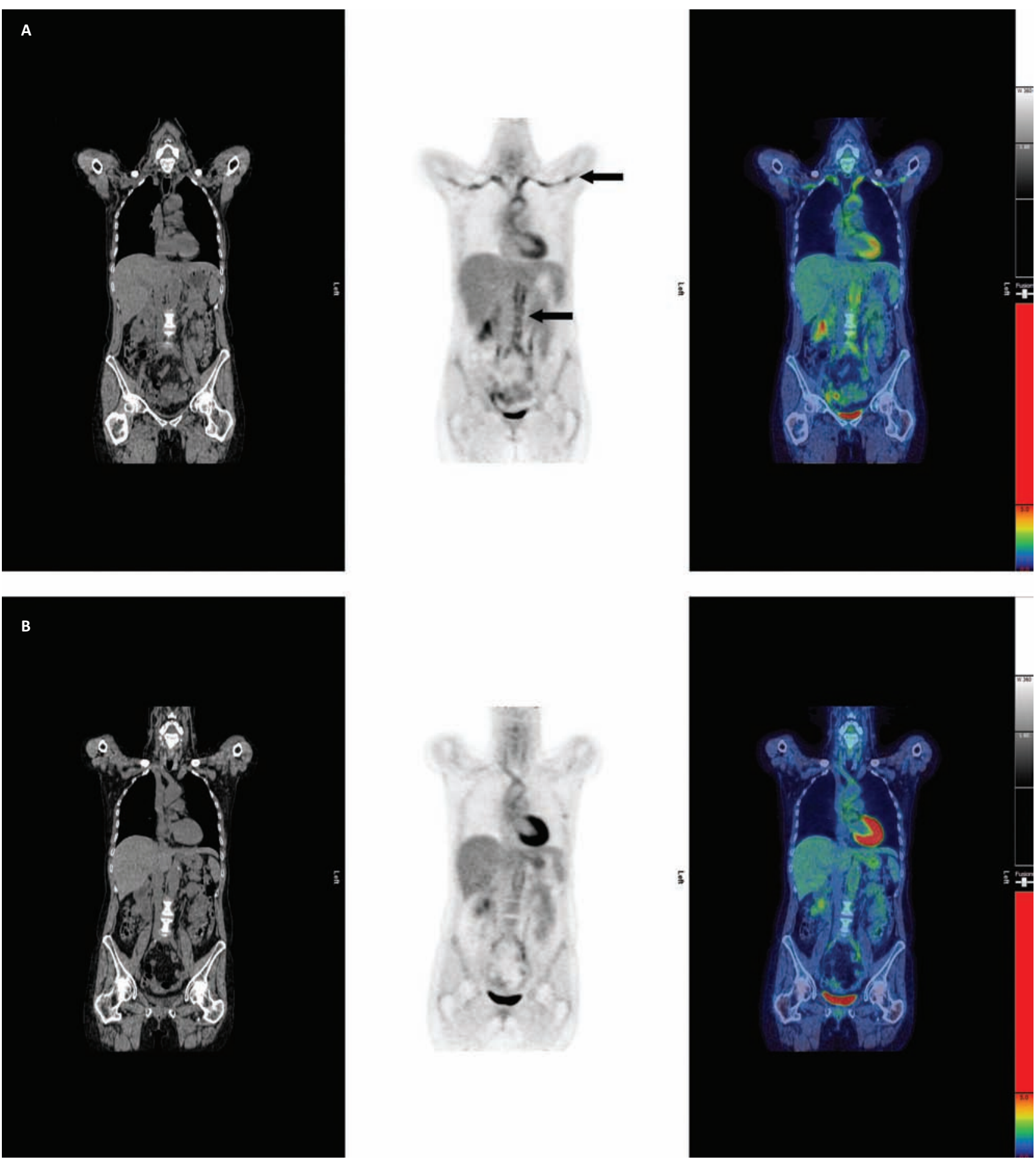

Fig 1. Takayasu's arteritis. A - 18F-FDG PET/CT scan coronal images of a patient presenting with weight loss, fevers, drenching night sweats and proximal muscle weakness. PET (left), CT (middle) and fused PET/CT (right) images are shown indicating increased uptake in large vessels, subclavian and abdominal aorta (arrows) compatible with the final diagnosis of Takayasu's giant cell arteritis. B - Repeat scan after 6 months with steroid therapy indicated reduction in uptake in the vessels. $\mathrm{CT}=$ computerised tomography; $\mathrm{FDG}=$ fluorodeoxyglucose; $\mathrm{PET}=$ positron emission tomography

provide contrast to more than one modality simultaneously. Some combined modality contrast agents are approaching clinical application, others are earlier in development. For example, radiopharmaceuticals generally rely on perfusion for delivery to their target; measurement of perfusion using
DCE MRI can, in principle, provide perfusion information, enabling perfusion effects to be disentangled from specific target effects. Of benefit to surgeons, the possibility of locating tumours, small metastases or sentinel lymph nodes prior to surgery using radionuclides is well-established. However, 


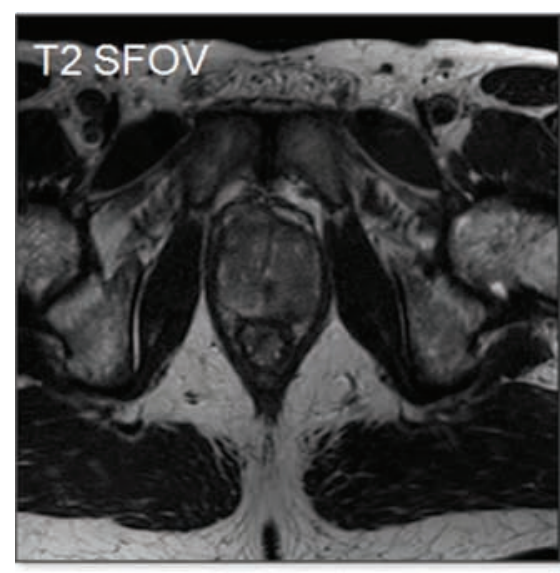

Fig 2. ${ }^{18} \mathrm{~F}$-choline PET/MRI axial images of a patient with Gleason $4+3$ prostate cancer. T2-weighted images (T2 SFOV) show a small area of low signal in the left posterior peripheral zone. There is restricted diffusion at this site on the ADC images, increased choline uptake on the fused T2/FDG PET image and increased blood flow compared to the normal prostate and normal muscle on the T1 DCE magnetic resonance images. $A D C=$ apparent diffusion coefficient; $\mathrm{DCE}=$ dynamic contrast enhanced; FDG = fluorodeoxyglucose; $\mathrm{MRI}=$ magnetic resonance imaging; $\mathrm{PET}=$ positron emission tomography; SFOV = small field of view
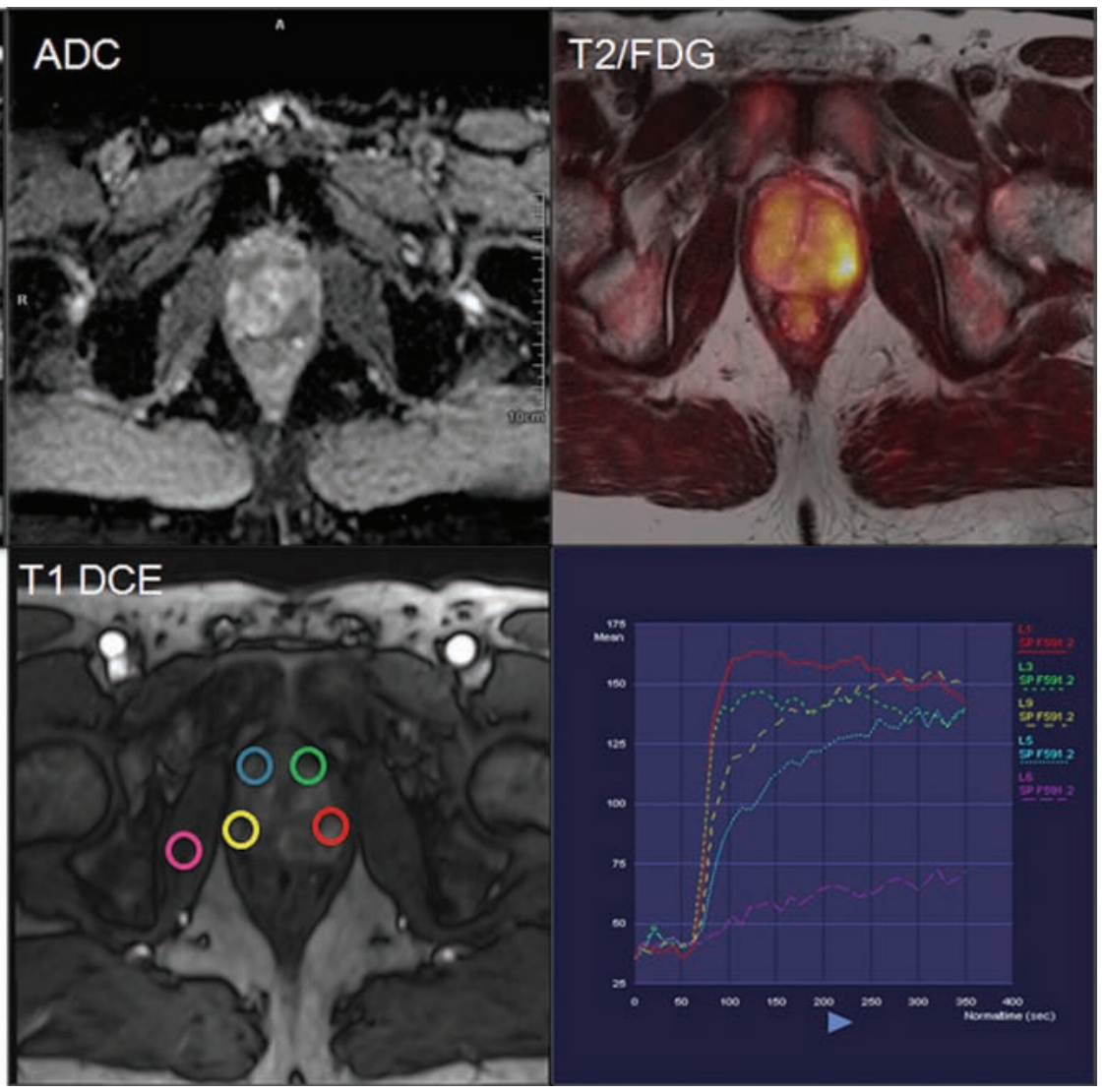

there is potential advantage to be gleaned by extending these imaging applications into the operating theatre by combining with fluorescent contrast agents with visible or near-infrared fluorophores to facilitate visualisation during surgery, for example to locate small tumours or to eliminate guesswork from delineation of tumour margins. ${ }^{12}$ An example of this approach is in the intra-operative detection of liver micrometastases with arginine-glycine-aspartic acid conjugated with indocyanine green mesoporous silica nanoparticles using a near-infrared fluorescent probe. ${ }^{13}$ A question to be resolved in the next few years is under what circumstances the contrast agents for each modality are better combined into one, or administered separately. The combination of multiple modality contrast into a single agent is challenging but an example is the recent development of superparamagnetic iron oxide particles, which provide negative MRI contrast radiolabelled with positron ${ }^{14}$ or gamma ${ }^{15}$ emitting radionuclides and indeed incorporating optical contrast as well. ${ }^{16}$ A potential application of such particles is locating sentinel lymph nodes (eg in breast cancer or melanoma) by taking advantage of the sensitivity offered by the radionuclide, while overcoming its poor resolution by simultaneously imaging with MRI. This can provide not only superior anatomical location but also, potentially, information about the pathological status of the node prior to surgery (eg to what extent has normal lymph node tissue been replaced by tumour). ${ }^{14}$ Optical contrast too can be incorporated into the superparamagnetic iron oxide particles to offer the surgeon easier intraoperative identification of the nodes. ${ }^{17}$
One advantage of combining a radionuclide and an optical tracer in the same molecule or entity is the ability to link whole body biodistribution information obtained by PET to microscopic cellular pathological information obtained in tissue samples. Microscopic visualisation of which cell types are taking up the tracer can greatly assist interpretation of PET scans in terms of the biology of the disease, a concept that has been shown in a preclinical breast cancer model expressing the human sodium iodide symporter fused to a red fluorescent protein, enabling radionuclide and fluorescent imaging to cross validate each other and provide improved sensitivity. ${ }^{18}$ Two separate contrast agents cannot do this with confidence. 'Smart' MR contrast agents able to change signal strength in response to a disease-related change in the microenvironment can in principle provide information about that microenvironment (eg $\mathrm{pH}$ change, calcium ion concentration, hypoxia). However, this information cannot easily be distinguished from the influence of local concentration of the MR contrast agent on signal strength. By incorporating a PET radionuclide into the contrast agent in a known fraction of molecules, the local concentration in tissue can be inferred from PET, ${ }^{19}$ thus providing the necessary calibration needed to make smart MRI a reality.

\section{Conclusions}

PET/CT is now established in clinical practice for oncologic and non-oncological applications. Improvement and development of scanner hardware has allowed faster acquisitions and 
wider application. PET/MRI offers potential improvements in diagnostic accuracy and patient acceptability but clinical applications are still being developed. A range of new radiotracers and non-radioactive contrast agents is likely to lead to a growth in hybrid molecular imaging applications that will enable better characterisation of disease processes.

\section{Conflicts of interest}

GC and SB receive research funding from Siemens Healthcare. SB receives research funding from Astra Zeneca and Hermes Medical Solutions.

\section{Acknowledgements}

The authors acknowledge financial support from the Department of Health via the National Institute for Health Research (NIHR) comprehensive Biomedical Research Centre awards to Guy's and St Thomas' NHS Foundation Trust in partnership with King's College London and the King's College London/University College London Comprehensive Cancer Imaging Centre funded by CRUK and EPSRC in association with the MRC and Department of Health (England).

\section{References}

1 Scarsbrook AF, Barrington SF. PET-CT in the UK: current status and future directions. Clin Radiol 2016;71:673-90.

2 Radford J, Illidge T, Counsell N et al. Results of a trial of PETdirected therapy for early-stage Hodgkin's lymphoma. N Engl J Med 2015;372:1598-607.

3 Johnson PWM, Federico M, Kirkwood AA et al. Adapted treatment guided by interim PET-CT scan in advanced Hodgkin lymphoma. N Engl J Med 2016;374:2419-29.

4 Royal College of Physicians, Royal College of Physicians and Surgeons of Glasgow, Royal College of Physicians of Edinburgh, Royal College of Radiologists, British Nuclear Medicine Society, Administration of Radioactive Substances Advisory Committee. Evidence-based indications for the use of PET-CT in the United Kingdom 2016. London: Royal College of Radiologists, 2016.

5 Freeman LM, Blaufox MD (eds). Theranostics. Semin Nucl Med 2012;42:145-218

6 Bonifacio G, Zamboni G. Brain imaging in dementia. Postgrad Med J 2016;92:333-40.

7 Leach MO, Brindle KM, Evelhoch JL et al. Assessment of antiangiogenic and antivascular therapeutics using MRI: recommendations for appropriate methodology for clinical trials. Br J Radiol 2003;76:S87-91.
8 Padhani AR, Koh DM, Collins DJ. Whole-body diffusion-weighted MR imaging in cancer: current status and research directions. Radiology 2011;261:700-18.

9 Spick C, Herrmann K, Czernin J. 18F-FDG PET/CT and PET/MRI perform equally well in cancer: evidence from studies on more than 2,300 patients. J Nucl Med 2016;57:420-30.

10 Messiou C, Kaiser M. Whole body diffusion weighted MRI-a new view of myeloma. Br J Haematol 2015;171:29-37.

11 Lindenberg L, Ahlman M, Turkbey B, Mena E, Choyke P. Advancement of MR and PET/MR in prostate cancer. Semin Nucl Med 2016;46:536-43.

12 van Dam GM, Ntziachristos V. Current concepts and future perspectives on intraoperative fluorescence imaging in cancer: clinical need. Curr Med Imaging Rev 2012;8:233-43.

13 Zeng C, Shang W, Wang K et al. Intraoperative identification of liver cancer microfoci using a targeted near infrared fluorescent probe for imaging-guided surgery. Sci Rep 2016;6:21959.

14 Torres Martin de Rosales R, Tavaré R, Paul RL et al. Synthesis of ${ }^{64} \mathrm{Cu}(\mathrm{II})$-bis (dithiocarbamate-bisphosphonate) and conjugation with superparamagnetic iron oxide nanoparticles: in vivo evaluation as dual-modality PET-MRI agent. Angew Chem Int Ed 2011;123:5623-7.

15 Torres Martin de Rosales R, Tavaré R, Glaria A et al. ${ }^{99 \mathrm{~m}} \mathrm{Tc}$ Bisphosphonate-iron oxide nanoparticle conjugates for dualmodality biomedical imaging. Bioconjugate Chem 2011;22:45565.

16 Cui X, Green MA, Blower PJ et al. $\mathrm{Al}(\mathrm{OH})_{3}$ Facilitated synthesis of water-soluble, magnetic, radiolabelled and fluorescent hydroxyapatite nanoparticles. Chem Commun 2015;51:9332-5.

17 van den Berg NS, Nynke S, Brouwer OR et al. Multimodal surgical guidance during sentinel node biopsy for melanoma: combined gamma tracing and fluorescence imaging of the sentinel node through use of the hybrid tracer Indocyanine Green-Tc-99mnanocolloid. Radiology 2015;275:521-9.

18 Fruhwirth GO, Diocou S, Blower PJ, Ng T, Mullen GE. A wholebody dual-modality radionuclide optical strategy for preclinical imaging of metastasis and heterogeneous treatment response in different microenvironments. J Nucl Med 2014;55:686-94.

19 Frullano L, Catana C, Benner T, Sherry AD, Caravan P. Bimodal MR-PET agent for quantitative pH imaging. Angew Chem Int Ed 2010;49:2382-4.

Address for correspondence: Professor Gary Cook, PET Centre, St Thomas' Hospital, Westminster Bridge Road, London SE1 7EH, UK.

Email: gary.cook@kcl.ac.uk 